\title{
Occult periprosthetic fractures of the acetabulum in THA using an elliptic cup design have no adverse impact on outcome
}

\author{
Thomas Vincent HäLler, Claudio Dora, Pascal Schenk, Patrick Oliver ZingG \\ From the Departement of Orthopaedics, Balgrist University Hospital, University of Zürich, Switzerland
}

Occult periprosthetic fractures have been defined as a fracture only visible on postoperative CT scans but not on postoperative plain radiography after an uneventful surgery without intraoperative fracture. The fracture rate for hemispherical and peripheral self-locking cups has been described as $8.4 \%$. We retrospectively analyzed postoperative $\mathrm{CT}$ scans after primary THA to clear the question whether such occult periprosthetic fractures of the acetabulum require particular treatment strategy.

Between 2014 and 2018 we identified 115 CT scans of 114 patients after primary cementless THA with elliptical cups using a direct anterior approach. The CT scans were obtained as part of other investigations. Localization of the fracture, patients demographics, clinical (WOMAC, Harris Hip Score) and radiological outcome were analyzed.

Fracture and non-Fracture group were compared with regard to demographics and short-term outcome after 1 year.

Four occult fractures $\mathbf{( 3 . 5 \% )}$ were identified. Three fractures involved the posterior wall. All patients had an uneventful routine postoperative rehabilitation. Patients with occult fractures showed similar postoperative HHS and WOMAC scores at 3 (HHS p = 0.576, WOMAC $p=0.128$ ) and 12 (HHS $p=0.479$, WOMAC $p=0.588)$ months. There were no cup loosening nor radiolucent lines at latest follow-up (mean FU 22 months, range 12-34 months).

There was no third party funding in this study. Each author certifies that he or she has no commercial associations (e.g. consultancies, stock ownership, equity interest, patent/ licensing arrangements, etc.) that might pose a conflict of interest in connection with the submitted article. All coauthors contributed substantially to the present study.
Clinical and radiological short-term outcome was not impaired by the occurrence of an occult periprosthetic fracture of the acetabulum. The incidental detection of an occult periprosthetic fracture of the acetabulum does not seem to oblige the surgeon to adapt the postoperative regime.

Keywords : Periprosthetic fracture ; acetabular fracture ; periprosthetic acetabular fracture ; total hip arthroplasty ; clinical outcome.

\section{INTRODUCTION}

Perioperative fractures of the femur or the acetabulum during THA are known complications (1-6). Intraoperative fractures of the acetabulum during

Thomas Vincent Häller ${ }^{1}$, M.D.

Claudio Dora ${ }^{1,2}$, M.D.

- Pascal Schenk ${ }^{1 *}$, M.D.

- Patrick Oliver Zingg ${ }^{1 *}$, M.D.

${ }^{1}$ Balgrist University Hospital, Departement of Orthopaedics, Forchstrasse 340, CH-8008 Zürich, Switzerland.

${ }^{2}$ Schulthess Klinik, Lengghalde 2, CH-8008 Zürich, Switzer-

land.

* Contributed equally as senior authors.

Correspondence : Dr. med. Thomas Vincent Häller, Universitätsklinik Balgrist, Forchstrasse 340, CH-8008 Zürich. Phone :

+414438657 85, Fax: +41443861290.

Email : thomas.haeller@ksb.ch

- 2021, Acta Orthopædica Belgica.

Acta Orthopædica Belgica, Vol. 87 - 2 - 2021 
primary THA are ascribable to the use of pressfit uncemented acetabular components, which has become more frequent during the past 20 years $(4,7,8)$. Intraoperatively recognized fractures of the acetabulum are a rare event (9-12). Their rate has been reported as $0.4 \%$ using plain radiographs (10). In contrast, periprosthetic fractures of the femur using cementless components in primary THA are much more frequent ranging from $2.95 \%$ to $27.8 \%$ $(4,13)$.

On plain radiographs a periprosthetic fracture of the acetabulum may not be visible immediately; MRI or CT frequently is required to confirm the suspicion of such a fracture (14).

Occult periprosthetic fractures have been defined as a fracture only visible on postoperative CT images but not on postoperative plain radiography after an uneventful surgery without intraoperative fracture (14). In patients with unusual groin pain during first postoperative weeks or as an incidental finding in computed tomography for other indications, such fractures occasionally are diagnosed $(14,15)$. In a recently published study the rate of such occult fractures was reported as $8.4 \%$ for mainly hemispheric and peripheral self-locking (PSL) cup design. There were only 3 elliptical cups and clinical outcome was not described (14).

The aim of this study was to investigate occult periprosthetic fractures of the acetabulum using elliptic cups with regard to location and the radiological and clinical outcome.

\section{MATERIALS AND METHODS}

We investigated a cohort of 114 consecutive patients that underwent primary cementless THA with postoperative CT scans in context of other studies on 3 dimensional planning (BASEC no 2012-0064) and on occult periprosthetic femoral shaft fractures (BASEC no 2012-0162) between May 2014 and March 2018 at our institution. The total number of THA during this period was 1338. All patients gave their written consent. The postoperative CT scan was performed between one and twelve weeks after surgery since it was not the actual aim of all of these CT scans to rule out a periprosthetic fracture.

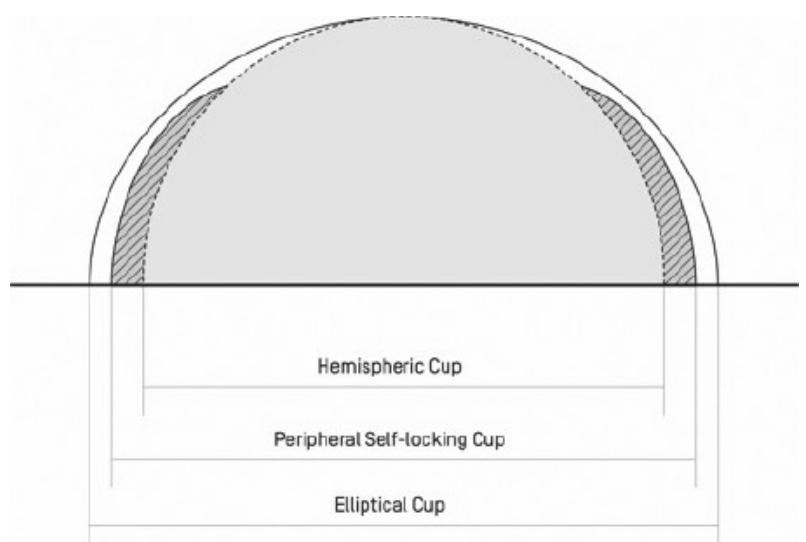

Figure 1. - Schematic depiction of hemispheric, peripheral self locking and elliptic cup designs. Elliptical cups have the form of a compressed circle and a larger diameter at the periphery. Peripheral self locking cups have a rim which enlarges the diameter at the periphery.

The study population comprised of 114 patients (115 hips ; 71 males and 43 females). The mean age was $59 \pm 9$ years (range, 38-83 years) and the mean BMI was $26.6 \pm 4.4 \mathrm{~kg} / \mathrm{m}^{2}$ (range $16.7-36.8 \mathrm{~kg} / \mathrm{m}^{2}$ ).

According to the categorization of acetabular components (Figure 1) in true hemispheric, peripheral self-locking (PSL) cups and elliptical cups, all our acetabular components were elliptic. All of the cups did not have preassembled cup and inlay, so they were classified as elliptic modular.

The following cups (all titanium alloy) were involved: April® $(\mathrm{n}=65)$ (Symbios Orthopédie SA, Yverdon-les-bains, Switzerland), Versafit CC trio ${ }^{\circledR}(\mathrm{n}=34)$ (Medacta International, Castel San Pietro, Switzerland) and Fitmore ${ }^{\circledR}(\mathrm{n}=16)$ (Zimmer-Biomet, Warsaw, IN, USA).

Indication for surgery included osteoarthritis (OA) in 111 hips and osteonecrosis of the femoral head in 3 patients. Preoperative planning was performed using 2D radiographic planning with MediCAD software (MediCAD Hectec GmbH, Altdorf bei Landshut, Germany). None of the hips received additional dome screw fixation during surgery. The surgeries were carried out by the surgical team, including senior surgeons or trainees supervised by senior surgeons. THA was performed with the patient supine on a traction table under regional or general anesthesia using a minimally invasive direct anterior approach (DAA) $(16,17)$. The technique of 
acetabular preparation was sized reaming in all cases. We planned the position of the cup at the level of the true acetabular fossa. The angle of the cup was aimed at an inclination of $40^{\circ}$ with $20^{\circ}$ of anteversion. Our routine postoperative regime after primary THA allows full weight bearing with crutches for two weeks postoperatively and patients start physiotherapy in the third postoperative week with the goal to get off crutches depending on muscle capacity. All patients were treated according to this protocol.

On the 115 available CT scans (Philips Brilliance 64 CT®; Koninklijke Philips NV, Amsterdam, Netherlands or the Siemens Somatom Definition AS 64® CT; Siemens AG, Berlin, Germany) the location of occult periprosthetic fractures of the acetabulum was assessed according to the modified classification of Callaghan (18) which was used by Hasegawa et al. (14) (1: medial wall, 2 : posterior wall, 3 : superolateral wall, 4 : anterior wall, 5 : other). All postoperative CT images were reviewed by a musculoskeletal radiologist.

In addition to the CT scans, all patients routinely had plain radiographs (antero-posterior pelvic and cross-table lateral views) preoperatively, immediately after surgery and at 3 and 12 months
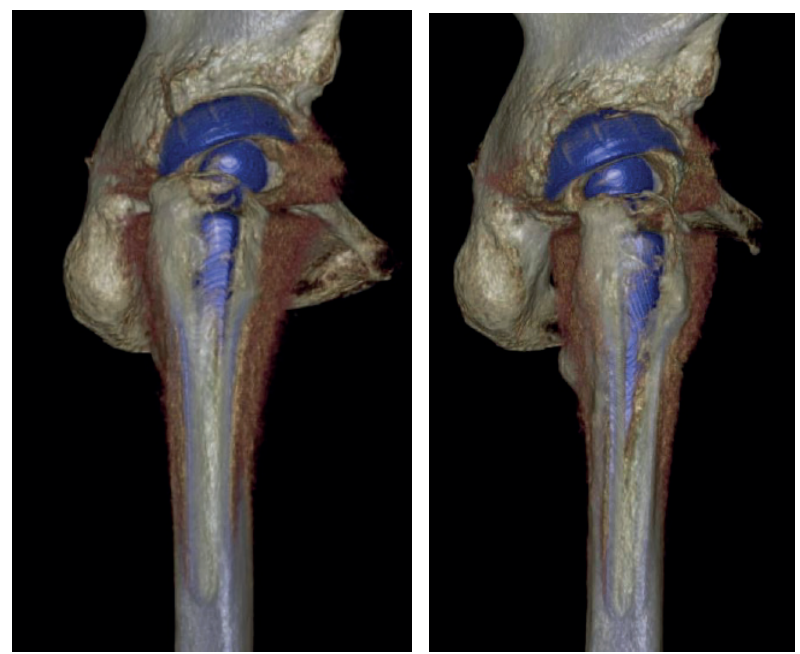

Figure 2a. - 3D CT recon- Figure 2b. - Same patient struction of the pelvis 2 days as in Figure 2a), 3 months postoperatively showing a frac- later. The fracture is completure of the posterior wall. tely consolidated and no dislocation of the cup was observed.

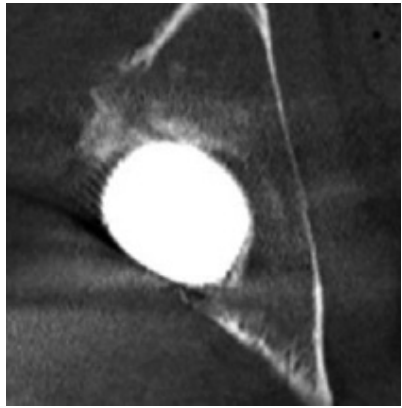

Figure 2c. - CT of the same patient than $2 \mathrm{a}$ ), sagittal view.

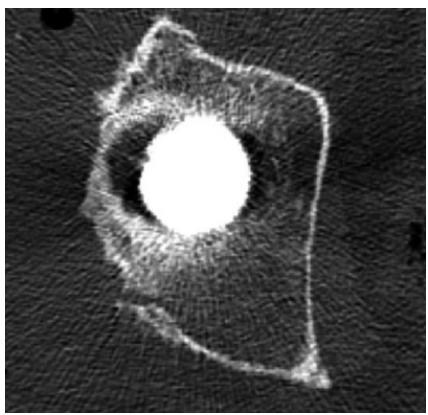

Figure 2e. - CT axial view.

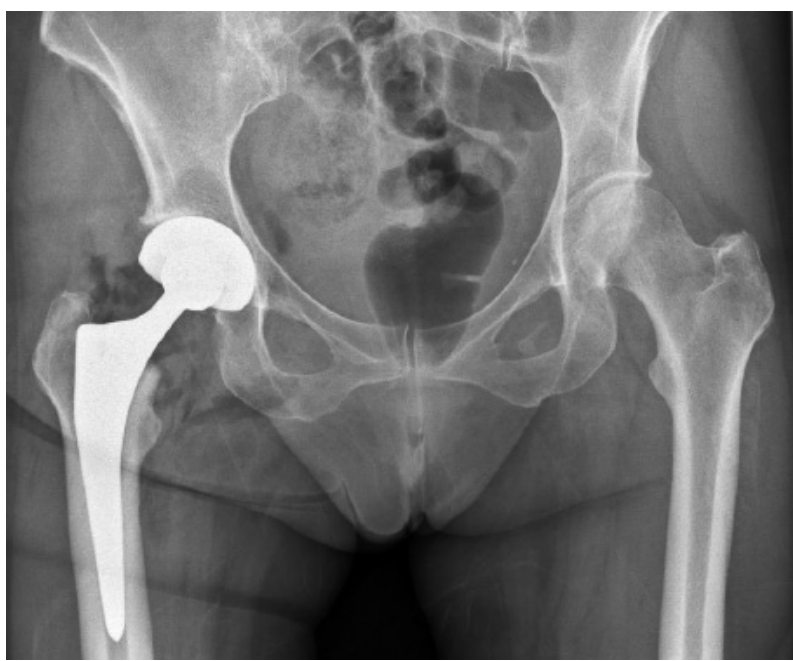

Figure $2 f$. - Same patient, postoperative plain radiograph ap.

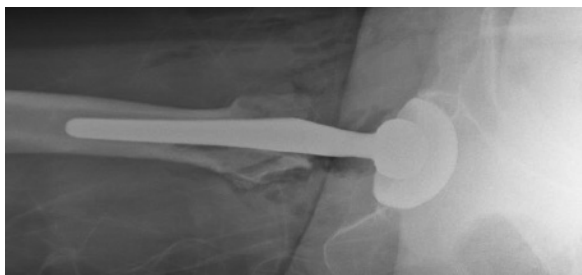

Figure 2g. - Same patient postoperative radiograph axial. 
postoperative. Plain radiographs after 3 and 12 months of all occult fractures were evaluated for radiolucent lines in the periacetabular zones according to DeLee and Charnley (19) or component dislocation.

With regard to the clinical outcome the Western Ontario and McMasters Universities Arthritis Index (WOMAC) score (20) and Harris Hip Score (21) (HHS), routinely documented at 3 and 12 months after surgery, were retrieved from the electronic medical chart. The clinical scores of the patients that sustained an occult periprosthetic fracture of the acetabulum (fracture group) were compared to those without (non-fracture group).

The study was approved by the local ethics committee (BASEC no 2018-01925).

Statistical analysis was performed using SPSS ${ }^{\circledR}$ version 23 (IBM, Armonk, USA). The KolmogorovSmirnov-Test was used analyzing the two groups for normal distribution. The Mann-Whitney U Test was performed to compare continuous data between the two groups. The statistical analyses defined a probability of 0.05 or less as significant.

\section{RESULTS}

There were 4 occult periprosthetic fractures of the acetabulum in our collective of 115 hips (3.5\%). In the subgroup receiving the April ${ }^{\circledR} \operatorname{cup}(n=65)$ the rate of occult periprosthetic fractures was $1.5 \%$ (1 fracture). Using the Versafit CC trio ${ }^{\circledR} \operatorname{cup}(n=34)$ the rate was $5.9 \%$ ( 2 fractures). Using the Fitmore ${ }^{\circledR}$ cup $(n=16)$ the rate was $6.3 \%$ ( 1 fracture).

Fracture localization : The most frequent fracture location was the posterior wall (zone 2), which was seen in 3 cases and one fracture involved the anterior wall (Zone 4). An example of an occult periprosthetic posterior wall fracture is shown in Figure 2.

No fracture lines or sclerosis zone was seen on any of the follow up plain radiographs. At latest radiographic follow up (mean 22 (range 12-34) months) there were no periprosthetic radiolucent lines or secondary component dislocation.

Clinical outcome: There was no significant difference of the HHS and WOMAC score at 3 (HHS $p=0.576$, WOMAC $p=0.128$ ) and 12 (HHS
Table I. — Patient related outcome scores

\begin{tabular}{|l|c|c|c|c|}
\hline \multirow{2}{*}{} & \multicolumn{2}{|c|}{$\begin{array}{c}\text { 3 months } \\
\text { postoperatively }\end{array}$} & \multicolumn{2}{c|}{$\begin{array}{c}\text { 12 months } \\
\text { postoperatively }\end{array}$} \\
\cline { 2 - 5 } & HHS & WOMAC & HHS & WOMAC \\
\hline $\begin{array}{l}\text { Occult } \\
\text { fracture } \\
(\mathbf{n = 4 )}\end{array}$ & $96( \pm 3.6)$ & $2.2( \pm 1.4)$ & $97( \pm 2.1)$ & $1.4( \pm 1.8)$ \\
\hline $\begin{array}{l}\text { No occult } \\
\text { fracture } \\
(\mathbf{n = 1 1 1})\end{array}$ & $90( \pm 12.3)$ & $1.2( \pm 1.3)$ & $94( \pm 9.8)$ & $0.8( \pm 1.0)$ \\
\hline p-value & 0.576 & 0.128 & 0.479 & 0.588 \\
\hline
\end{tabular}

Patient related outcome scores (HHS and WOMAC) for both groups. Mean (SD)

$p=0.479$, WOMAC $p=0.588)$ months after surgery between the two groups (Table I). The BMI did not show any significance between patients sustaining an occult fracture and those without $(\mathrm{p}=0.555)$. Patients with an occult periprosthetic fracture of the acetabulum did not have any other diseases that could debilitate their bone quality such as rheumatic arthritis or osteoporosis.

\section{DISCUSSION}

In contrary to intraoperative periprosthetic fractures of the femur, the acetabulum is less often afflicted. The diagnosis of intraoperative periprosthetic acetabular fractures is demanding because the periacetabular area has a complex shape and the bone is covered by dense soft tissue. However, from our point of view the occult periprosthetic acetabular fractures have to be separated from intraoperative detected or with a plane radiograph diagnosed periprosthetic acetabular fractures. We believe that in occult fractures the support of the acetabular ring is still enough to guarantee the anteroposterior press fit and therefore the stability of the cup. Leading to the assumption, that patients with an occult periprosthetic fracture of the acetabulum do not need partial weight bearing or any further interventions diverge from the routine postoperative rehabilitation program. As a postoperative CT after THA can have several indications (22) and the diagnosis of an occult periprosthetic fracture of the acetabulum will be made sometimes incidentally this will lead to the question on how to treat these fractures. 
Since we use elliptic cup designs at our institution we decided to evaluate the rate and location of occult periprosthetic fractures of the acetabulum in our collective and focus our attention not only on the radiographic follow-up but also on the clinical outcome. To our knowledge this is the first study looking at occult periprosthetic fractures of the acetabulum with the use of elliptical cups in a larger collective using pre- and postoperative CT scans and evaluating the clinical and radiological outcome. Our goal was not to evaluate predictive factors for such occult fractures since the number of cups of each brand would have been too low to allow for any multivariant regression analysis.

Our rate of occult periprosthetic fractures of the acetabulum was $3.5 \%$ ( 4 of 115). This is lower than the unexpectedly high rate of $8.4 \%$ reported by Hasegawa et al. (14). They could confirm that these minor periprosthetic fractures occurred during press-fit impaction. They used mostly peripheral self-locking (PSL) and hemispheric cups and only 3 elliptic cups, which was the only cup design in the present study. The different cup design may be an explanation for this difference in fracture rates.

Our fractures where located mainly in the posterior wall (3 out of 4). Most of the fractures in the study of Hasegawa et al. were located in the superolateral wall (37\%).(14) The different locations may be explained by the different approaches: Whilst we used a DAA, Hasegawa used primarily a posterolateral approach. The direction of cup insertion could explain these different locations.

Clinical and radiological follow up did not show any differences between the fracture and the control group. The radiological assessment did not reveal radiolucent lines or cup migration at the latest follow up.

Regarding the clinical follow up, patients with an occult periprosthetic fracture of the acetabulum did not report any unusual postoperative pain or problems and their HHS and WOMAC score were not significantly different from those without an occult fracture. The patients with a fracture did not receive any additional treatment, especially no partially weight bearing. This makes us conclude that patients who are sustaining an occult periprosthetic fracture of the acetabulum do not need adaption of the rehabilitation protocol. However, a recent study from 2018 found that occult periprosthetic fractures of the central wall had to be revised in the early and mid-term postoperative phase since implant survival may be impaired (22). They inserted mostly PSL or hemispherical cups and only one elliptical cup and used a DAA and lateral transgluteal approach. Because none of our fractures were located in the central wall, we can neither deny nor confirm this statement. In the present study occult periprosthetic fractures in of the posterior (Zone 2) or anterior wall (Zone 4) do not lead to early implant failure.

There are several limitations with this study: First, we only diagnosed 4 occult periprosthetic fractures of the acetabulum, which makes the size of the fracture group rather small and limits the statistical power with regard to the clinical outcome. Second, the interval to the postoperative CT scan was rather long with 12 weeks maximum in a subgroup of 65 patients. The other patients obtained their CT in the first postoperative week. Nevertheless, we diagnosed one fracture in the group which had their CT 12 weeks postop, which leads us to the assumption that an occult fracture should still be visible after this time. Anyhow this could have led to an underreporting of the fracture rate.

Third, we are reporting about short-term outcome only. However, we consider this as sufficient looking at bony union, cup loosening or dislocation associated to an intraoperative fracture. Since there were no pathological radiological findings, we do not expect the clinical short-term outcome to alter on a longer course.

\section{CONCLUSION}

We conclude that the incidental detection of an occult periprosthetic fracture of the acetabulum does not seem to oblige the surgeon to adapt the postoperative regime, since clinical and radiological outcome is not impaired.

\section{Acknowledgements}

We are indebted to Dr. med. Christoph Agten for reviewing the CT scans, Ms. Nadja Rauer for her 
support with the artwork and all colleagues of our Orthopedic Department and of the Unit for Clinical and Applied Research (UCAR) for their help.

\section{REFERENCES}

1. Thillemann TM, Pedersen AB, Johnsen SP, Soballe K. Inferior outcome after intraoperative femoral fracture in total hip arthroplasty: outcome in 519 patients from the Danish Hip Arthroplasty Registry. Acta Orthop. 2008 ; 79(3) : 327-34.

2. Davidson D, Pike J, Garbuz D, Duncan CP, Masri BA. Intraoperative periprosthetic fractures during total hip arthroplasty. Evaluation and management. J Bone Joint Surg Am. 2008 ; 90(9) : 2000-12.

3. Pike J, Davidson D, Garbuz D, Duncan CP, O'Brien PJ, Masri BA. Principles of treatment for periprosthetic femoral shaft fractures around well-fixed total hip arthroplasty. J Am Acad Orthop Surg. 2009 ; 17(11) : 677-88.

4. Berry DJ. Epidemiology : hip and knee. Orthop Clin North Am. $1999 ; 30(2): 183-90$.

5. Berend KR, Lombardi AV, Jr. Intraoperative femur fracture is associated with stem and instrument design in primary total hip arthroplasty. Clin Orthop Relat Res. 2010 ; 468(9) : 2377-81.

6. Berend KR, Lombardi AV, Jr., Mallory TH, Chonko DJ, Dodds KL, Adams JB. Cerclage wires or cables for the management of intraoperative fracture associated with a cementless, tapered femoral prosthesis : results at 2 to 16 years. J Arthroplasty. 2004 ; 19(7 Suppl 2) : 17-21.

7. Troelsen A, Malchau E, Sillesen N, Malchau H. A review of current fixation use and registry outcomes in total hip arthroplasty : the uncemented paradox. Clin Orthop Relat Res. 2013 ; 471(7) : 2052-9.

8. Akiyama H, Hoshino A, Iida H, Shindo H, Takakura Y, Miura H, et al. A pilot project for the Japan arthroplasty register. J Orthop Sci. 2012 ; 17(4) : 358-69.

9. Peterson CA, Lewallen DG. Periprosthetic fracture of the acetabulum after total hip arthroplasty. J Bone Joint Surg Am. 1996 ; 78(8) : 1206-13.
10. Haidukewych GJ, Jacofsky DJ, Hanssen AD, Lewallen DG. Intraoperative fractures of the acetabulum during primary total hip arthroplasty. J Bone Joint Surg Am. 2006 ; 88(9) : 1952-6.

11. Curtis MJ, Jinnah RH, Wilson VD, Hungerford DS. The initial stability of uncemented acetabular components. J Bone Joint Surg Br. 1992 ; 74(3) : 372-6.

12. Chitre A, Wynn Jones H, Shah N, Clayson A. Complications of total hip arthroplasty : periprosthetic fractures of the acetabulum. Curr Rev Musculoskelet Med. 2013 ; 6(4) : 357-63.

13. Jensen JS, Retpen JB. Failures with the Judet noncemented total hip. Acta Orthop Scand. 1987 ; 58(1) : 23-6.

14. Hasegawa K, Kabata T, Kajino Y, Inoue D, Tsuchiya $H$. Periprosthetic Occult Fractures of the Acetabulum Occur Frequently During Primary THA. Clin Orthop Relat Res. 2017 ; 475(2) : 484-94.

15. Sharkey PF, Hozack WJ, Callaghan JJ, Kim YS, Berry DJ, Hanssen AD, et al. Acetabular fracture associated with cementless acetabular component insertion : a report of 13 cases. J Arthroplasty. 1999 ; 14(4) : 426-31.

16. Moreau P. Minimally invasive total hip arthroplasty using Hueter's direct anterior approach. Eur J Orthop Surg Traumatol. $2018 ; 28(5)$ : 771-9.

17. Hueter C. Grundriss der Chirurgie : F.C.W. Vogel ; 1883.

18. Callaghan JJ. Periprosthetic fractures of the acetabulum during and following total hip arthroplasty. Instr Course Lect. 1998 ; $47: 231-5$.

19. DeLee JG, Charnley J. Radiological demarcation of cemented sockets in total hip replacement. Clin Orthop Relat Res. 1976(121) : 20-32.

20. Klassbo M, Larsson E, Mannevik E. Hip disability and osteoarthritis outcome score. An extension of the Western Ontario and McMaster Universities Osteoarthritis Index. Scand J Rheumatol. 2003 ; 32(1) : 46-51.

21. Harris WH. Traumatic arthritis of the hip after dislocation and acetabular fractures : treatment by mold arthroplasty. An end-result study using a new method of result evaluation. J Bone Joint Surg Am. 1969 ; 51(4) : 737-55.

22. Dammerer D, Putzer D, Glodny B, Petersen J, Arrich F, Krismer M, et al. Occult intra-operative periprosthetic fractures of the acetabulum may affect implant survival. Int Orthop. 2018. 